\title{
Should we avoid colleagues in leisure time during the coronavirus disease 2019 (COVID-19) pandemic?
}

\author{
Lotta-Maria A. H. Oksanen MD ${ }^{1,2}$ (1), Enni Sanmark PhD, MD ${ }^{1,2}$ (1), Sampo A. Oksanen MSc Econ ${ }^{3}$ (1),

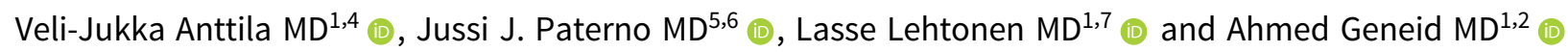 \\ ${ }^{1}$ Faculty of Medicine, University of Helsinki, Helsinki, Finland, ${ }^{2}$ Department of Otorhinolaryngology and Phoniatrics - Head and Neck Surgery, Helsinki University \\ Hospital, Helsinki, Finland, ${ }^{3}$ Nordic Healthcare Group, Helsinki, Finland, ${ }^{4}$ Inflammation Center, Helsinki University Hospital, Helsinki, Finland, ${ }^{5}$ Department of \\ Ophthalmology, Kuopio University Hospital, Kuopio, Finland, ${ }^{6}$ Faculty of Medicine, University of Eastern Finland, Kuopio, Finland and ${ }^{7}$ Diagnostic Center, \\ HUSLAB, Helsinki University Hospital, Helsinki, Finland
}

To the Editor- Coronavirus disease 2019 (COVID-19) is a significant occupational threat for healthcare workers (HCWs). ${ }^{1}$ The high number of infected HCWs has been explained with occupational exposure to severe acute respiratory coronavirus virus 2 (SARS-CoV-2). Hospitals have implemented infection control measures including proper personal protective equipment (PPE), universal masking in hospitals, and safety distance between coworkers to reduce the transmission. ${ }^{2,3}$ However, studies of HCW exposure to COVID-19 outside the workplace have not been published.

We examined HCW occupational and nonoccupational exposures to SARS-CoV-2 in tertiary-care hospitals (Uusimaa, Finland) during the first wave of the COVID-19 pandemic through July 15 , 2020. Of 1,072 HCWs enrolled in our study, 866 nurses, midwives, and doctors from the Helsinki University Hospital answered questionnaires by July 15, 2020, regarding their infection symptoms, workplace, exposure to COVID-19, and use of PPE. All participants presenting any COVID-19-related symptoms were tested using standard reverse-transcriptase polymerase chain reaction (RT-PCR) methods. ${ }^{4}$ Participants' medical histories were reviewed in July 2020 for SARS-CoV-2 RT-PCR and antibody results. The demographic backgrounds of the participants were similar to those of Helsinki University Hospital (HUS) personnel overall. ${ }^{5}$

All infected participants were contacted, and the results were confirmed and analyzed. COVID-19 exposure was divided into workplace exposure, outside the workplace exposure, or unknown exposure and further into colleague-related exposure, patientrelated exposure, or other exposure. The statistical analysis was performed using the Fisher exact test with SPSS version 27 software (IBM, Armonk, NY).

Overall, 41 participants had COVID-19 and presented either a SARS-CoV-2-positive RT-PCR or antibody test. Of these 41 HCWs, 22 were likely infected at a workplace and 15 were likely infected outside the workplace; the rest remained unclear. The source of infection was likely nonpatient exposure for 21 infected participants (51\%). The absolute risk for colleague-origin COVID-19 in

Author for correspondence: Lotta-Maria Adele Helena Oksanen, E-mail: lotta. oksanen@hus.fi

Cite this article: Oksanen L-MAH, et al. (2021). Should we avoid colleagues in leisure time during the coronavirus disease 2019 (COVID-19) pandemic? Infection Control \& Hospital Epidemiology, 42: 1413-1414, https://doi.org/10.1017/ice.2020.1320 this sample was $1.4 \%$, whereas overall regional risk was $0.3 \%{ }^{5}$ Also, 7 workplace-related infections (32\%) and 5 infections originating outside the workplace (33\%) were transmitted by colleagues.

In our sample, if the source of COVID-19 infection was not a patient, the infection was more likely to be from a colleague $(P<.001)$, and $42 \%$ of infections from a colleague occurred during leisure time. Notably, participants who treated COVID-19 patients (outside the ICU) had an increased risk for COVID-19 overall (OR, 2.6; $95 \% \mathrm{CI}, 1.4-5.2 ; P=.003)$, but even then, 5 of 26 COVID-19 cases $(19 \%)$ were transmitted by colleagues outside the workplace.

Although it may seem self-evident, meeting with other HCWs outside the workplace could introduce an independent risk for a COVID-19 infection, especially among those working with COVID-19 patients. Given the close work communities in health care, where social interactions at work and leisure are traditionally easily mixed, the risk of free-time interactions between workers who work with COVID-19 patients should be carefully considered. In this study, we were unable to provide enough evidence for the authoritative guidance to employees because the sample size was limited. Also, it is likely that some asymptomatic SARS-CoV-2-positive participants were not recognized. Nonetheless, it is imperative to observe social distance in collegial interaction in leisure time until there is an effective vaccine against COVID-19.

Acknowledgments. We thank Catharina Pomoell for her great work as a research nurse and Johan Sanmark for his support. We also thank all participants involved in this study and their help to find ways to ensure safe working environment.

Financial support. This work was supported by the Helsinki University Hospital Research Fund (grant no. Y1014KN025).

Conflicts of interest. All authors report no conflicts of interest relevant to this article.

\section{References}

1. Adams JG, Walls RM. Supporting the healthcare workforce during the COVID-19 global epidemic. JAMA 2020;323:1439-1440.

2. Chu DK, Akl EA, Duda S, et al. Physical distancing, face masks, and eye protection to prevent person-to-person transmission of SARS-CoV-2 and COVID-19: a systematic review and meta-analysis. Lancet 2020;395: 1973-1987.

3. Advice on the use of mask in the context of COVID-19. World Health Organization website. https://www.who.int/publications/i/item/advice-on-theuse-of-masks-in-the-community-during-home-care-and-in-healthcare-settings-

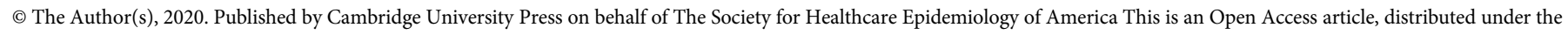

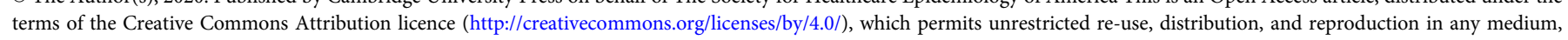
provided the original work is properly cited. 
in-the-context-of-the-novel-coronavirus-(2019-ncov)-outbreak. Published May 6, 2020. Accessed November 11, 2020.

4. Mannonen L, Kallio-Kokko H, Loginov R, et al. Comparison of two commercial platforms and a laboratory developed test for detection of SARS-CoV-2 RNA. medRxiv 2020. doi: 10.1101/2020.07.03.20144758.
5. Oksanen L, Sanmark E, Oksanen S, et al. Healthcare workers high COVID-19 infection rate: the source of infections and potential for respirators and surgical masks to reduce occupational infections. medRxiv 2020. doi: $10.1101 / 2020.08 .17 .20176842$.

\title{
Missed vaccine opportunities for Streptococcus pneumoniae and influenza in patients admitted during the coronavirus disease 2019 (COVID-19) pandemic
}

\author{
Anita Shallal MD ${ }^{1}$ (D), Rachel Kenney PharmD² and Allison Weinmann MBBS, FRACP, FSHEA ${ }^{3,4,5}$ \\ ${ }^{1}$ Division of Infectious Diseases, Department of Internal Medicine, Henry Ford Hospital, Detroit Michigan, ${ }^{2}$ Department of Pharmacy Services, Henry Ford \\ Hospital, Detroit, Michigan, ${ }^{3}$ Antimicrobial Stewardship, Division of Infectious Diseases, Henry Ford Health System, Detroit, Michigan, ${ }^{4}$ Office of Clinical Quality \\ and Safety, Henry Ford Health System, Detroit, Michigan and ${ }^{5}$ Department of Internal Medicine, Wayne State University, Detroit, Michigan
}

To the Editor-Detroit, Michigan, and its surrounding counties emerged as a hot spot for the coronavirus disease-2019 (COVID-19) pandemic in March and April of 2020. A number of hospitalized patients had numerous comorbidities, which increased the risk of acquiring the infection: age $>65$ years, heart or lung disease, lung disease, and diabetes. These comorbidities are also associated with other infections, including invasive Streptococcus pneumoniae infection. Our institution follows a standing order for nurses to vaccinate adults aged $>65$ years who meet the Advisory Committee on Immunization Practices (ACIP) criteria for the 23-valent pneumococcal polysaccharide vaccine (PPSV23) to reduce $S$. pneumoniae infections ${ }^{1,2}$ and against influenza during flu season. During the COVID-19 pandemic surge, the pneumococcal vaccine and influenza vaccine nurse-driven protocols were determined to be nonessential on March 23, 2020, and April 2, 2020, respectively. In this study, we aimed to characterized missed vaccine opportunities among patients hospitalized with COVID-19 during this surge.

We performed a cross-sectional study of PCR-positive COVID19 patients admitted to an inner-city tertiary-care health system and discharged alive between the dates of March 23 and April 21,2020 . Patients under the age of 65 were excluded. Data were collected retrospectively and included patient age, gender, race, length of stay, comorbidities that would indicate a vaccine opportunity, prior vaccinations, and whether there was a vaccine opportunity for PPSV23 and influenza defined by ACIP indications. ${ }^{2}$ The indications included age $>65$ years, chronic lung disease, chronic kidney disease, cardiomyopathy or heart failure, human immunodeficiency virus (HIV), solid-organ malignancy or multiple myeloma, immunosuppressed (ie, on immunosuppressing drugs, long-term steroids, or solid-organ recipient), and other (eg, cochlear implant, cerebrospinal fluid leak, post-splenectomy,

Author for correspondence: Anita Shallal, E-mail: AShalla2@hfhs.org

PREVIOUS PRESENTATION: This abstract was presented as a poster presentation at ID Week 2020, October 21-24, 2020, in Boston, Massachusetts.

Cite this article: Shallal A, Kenney R, and Weinmann A. (2021). Missed vaccine opportunities for Streptococcus pneumoniae and influenza in patients admitted during the coronavirus disease 2019 (COVID-19) pandemic. Infection Control \& Hospital Epidemiology, 42: 1414-1415, https://doi.org/10.1017/ice.2020.1294 sickle-cell disease, or alcohol-use disorder). Vaccine history was evaluated using the electronic medical record (EMR) and Michigan Care Improvement Registry (MCIR). If there was a vaccine opportunity, we documented whether or not a vaccine was given prior to hospital discharge. In addition, the total numbers of vaccines given for the same periods in 2019 and 2020 were collected from the EMR for comparison. Descriptive analysis was utilized.

Overall, 100 patients over the age of 65 were included. The median age was 71 years; most patients (66\%) were of African American race; and $87 \%$ of patients received antibiotics during hospitalization. We identifed 52 patients as having an opportunity to receive PPSV23, and none patients received the vaccine. Furthermore, 37 patients were eligible to receive influenza vaccine, and none received the vaccine. The median length of stay was 4 days, and 18 patients were readmitted within 30 days. ${ }^{3,6}$ Additional results are summarized in Table 1. According to the EMR, the total numbers of pneumococcal vaccinations given per nurse-driven protocol at our institution were 238 for March, 216 for April, and 218 for May. However, these numbers dropped to 123 for March, 11 for April, and 29 for May, corresponding to a percentage decrease in the number of vaccinations given to between $48 \%$ and $94 \%$. Similarly, the total numbers of influenza vaccinations given per nurse-driven protocol at our institution were 399 for March, 228 for April, and 38 for May. For the same period in 2020, these numbers dropped to 316 for March, 14 for April, and 0 for May, corresponding to a percentage decrease again as high as $94 \%$.

Due to prioritization of potential staffing shortages and clustering nursing care, a nursing-by-designated-authority protocol for immunization was deemed nonessential during the COVID-19 pandemic surge. As a result, opportunities to vaccinate patients with pneumococcal and influenza vaccines were missed despite an average hospitalization of 4 days to provide the vaccine(s). Of the 52 patients who had an opportunity for pneumococcal vaccination, most $(67.3 \%)$ had $>1$ indication for PPSV23, with $7.7 \%$ having 4 or more indications. These patients, due to numerous comorbidities, are at high risk for severe pneumococcal disease. The Centers for Disease Control and Prevention (CDC) has offered guidance that during the pandemic, confirmed positive COVID-19 patients should defer routine immunization. ${ }^{3}$ Although a febrile

(C) The Author(s), 2020. Published by Cambridge University Press on behalf of The Society for Healthcare Epidemiology of America. This is an Open Access article, distributed under the terms of the Creative Commons Attribution licence (http://creativecommons.org/licenses/by/4.0/), which permits unrestricted re-use, distribution, and reproduction in any medium, provided the original work is properly cited. 